\title{
All Together Now: Impact of a Regionalization and Bedside Rounding Initiative on the Efficiency and Inclusiveness of Clinical Rounds
}

\author{
Kristin T. L. Huang, MD¹,3, Jacquelyn Minahan², Patricia Brita-Rossi, RN, MSN, MBA ${ }^{1}$, Patricia Aylward, RN, MSN¹, \\ Joel T. Katz, MD 2,3 , Christopher Roy, MD²,3, Jeffrey L. Schnipper, MD, MPH ${ }^{2,3}$, Robert Boxer, MD, PhD $2,3 *$
}

'Brigham and Women's Hospital, Boston, Massachusetts; 'Division of General Internal Medicine, Brigham and Women's Hospital, Boston, Massachusetts; ${ }^{3}$ Harvard Medical School, Boston, Massachusetts.

BACKGROUND: Attending rounds at academic medical centers are often disconnected from patients and team members who are not physicians. Regionalization of care teams may facilitate bedside rounding and more frequent interactions among doctors, nurses, and patients.

OBJECTIVE: We used time-motion analysis to investigate how regionalization of medical teams and encouragement of bedside rounds affect participants on rounds and rounding time.

DESIGN AND SETTING: We used pre-post analysis to study the effects of care redesign on teams' daily rounds on a general medicine service at an academic medical center.

PARTICIPANTS: Four general medical teams were evaluated before the intervention and 5 teams afterward.

INTERVENTIONS: General medical teams were regionalized to specific units, the admitting structure was changed to facilitate regionalization, and teams were encouraged to round bedside.
MEASUREMENTS: Primary outcomes included proportion of time each team member was present on rounds and proportion of bedside rounding time. Secondary outcomes included round duration and non-patient time during rounds.

RESULTS: Proportion of time the nurse was present on rounds increased from $24.1 \%$ to $67.8 \%(P<0.001)$, and proportion of total bedside rounding time increased from $39.9 \%$ to $55.8 \%(P<0.001)$. Mean total rounding time decreased from 3.0 hours to 2.4 hours $(P=0.01)$, despite a higher patient census.

CONCLUSIONS: Creating regionalized care teams and encouraging interdisciplinary bedside rounds increased the proportion of bedside rounding time and the presence of nurses on rounds while decreasing total rounding time. Journal of Hospital Medicine 2017;12:150-156. (C) 2017 Society of Hospital Medicine
Attending rounds at academic medical centers are often disconnected from patients and non-physician care team members. Time spent bedside is consistently less than one third of total rounding time, with observational studies reporting a range of $9 \%$ to $33 \%$ over the past several decades. ${ }^{1-8}$ Rounds are often conducted outside patient rooms, denying patients, families, and nurses the opportunity to participate and offer valuable insights. Lack of bedside rounds thus limits patient and family engagement, patient input into the care plan, teaching of the physical examination, and communication and collaboration with nurses. In one study, physicians and nurses on rounds engaged in interprofessional communication in only $12 \%$ of patient cases. ${ }^{1}$ Studies have found interdisciplinary bedside rounds have several benefits, including subjectively improved communication and teamwork between physicians and nurses; increased patient satisfaction, including feeling more cared for by the medical team; and decreased length of stay and costs of care..$^{2-10}$

However, there are many barriers to conducting interdisciplinary bedside rounds at large academic medical centers.

*Address for correspondence and reprint requests: Robert Boxer, MD, PhD, Brigham and Women's Hospital, 75 Francis St, PBB-B 412, Boston, MA 02115; Telephone: 617-278-0055; Fax: 617-278-6906; E-mail: rboxer@partners.org

Received: May 16, 2016; Revised: August 16, 2016; Accepted: September 11,2016

2017 Society of Hospital Medicine DOI 10.12788/jhm.2596
Patients cared for by a single medical team are often geographically dispersed to several nursing units, and nurses are unable to predict when physicians will round on their patients. This situation limits nursing involvement on rounds and keeps doctors and nurses isolated from each other. ${ }^{2}$ Regionalization of care teams reduces this fragmentation by facilitating more interaction among doctors, patients, families, and nursing staff.

There are few data on how regionalized patients and interdisciplinary bedside rounds affect rounding time and the nature of rounds. This information is needed to understand how these structural changes mediate their effects, whether other steps are required to optimize outcomes, and how to maximize efficiency. We used time-motion analysis (TMA) to investigate how regionalization of medical teams, encouragement of bedside rounding, and systematic inclusion of nurses on ward rounds affect amount of time spent with patients, nursing presence on rounds, and total rounding time.

\section{METHODS}

\section{Setting}

This prospective interventional study, approved by the Institutional Review Board of Partners HealthCare, was conducted on the general medical wards at Brigham and Women's Hospital, an academic 793-bed tertiary-care center in Boston, Massachusetts. Housestaff teams consist of 1 attending, 1 resident, and 2 interns with or without a med- 
ical student. Before June 20, 2013, daily rounds on medical inpatients were conducted largely on the patient unit but outside patient rooms. After completing most of a rounding discussion outside a patient's room, the team might walk in to examine or speak with the patient. A typical medical team had patients dispersed over 7 medical units on average, and over as many as 13 . As nurses were unit based, they did not consistently participate in rounds.

\section{Intervention}

In June 2013, as part of a general medical service care redesign initiative, the general medical teams were regionalized to specific inpatient units. The goal was to have teams admit patients predominantly to the team's designated unit and to have all patients on a unit be cared for by the unit's assigned team as often as possible, with an $85 \%$ goal for both. Toward those ends, the admitting structure was changed from a traditional 4-day call cycle to daily admitting for all teams, based on each unit's bed availability. ${ }^{11}$

Teams were also expected to conduct rounds with nurses, and a system for facilitating these rounds was established. As physician and nurse care teams were now geographically co-located, it became possible for residents and nurses to check a rounding sheet for the planned patient rounding order, which had been set by the resident and nurse-in-charge before rounds. No more than about 5 minutes was needed to prepare each day's order. The rounding sheet prioritized sick patients, newly admitted patients, and planned morning discharges, but patients were also always grouped by nurse. For example, the physician team rounded with the first nurse on all 3 of a nurse's patients, and then proceeded to the next group of 3 patients with the next nurse, until all patients were seen.

Teams were encouraged to conduct patient- and family-centered rounds exclusively at bedside, except when bedside rounding was thought to be detrimental to a patient (eg, one with delirium). After an intern's bedside presentation, which included a brief summary and details about overnight events and vital signs, the concerns of the patient, family, and nurse were shared, a focused physical examination performed, relevant data (eg, laboratory test results and imaging studies) reviewed, and the day's plan formulated. The entire team, including the attending, was expected to have read new patients' admission notes before rounds. Bedside rounds could thus be focused more on patient assessment and patient/family engagement and less on data transfer.

Several actions were taken to facilitate these changes. Residents, attendings, nurses, and other interdisciplinary team members participated in a series of focus groups and conferences to define workflows and share best practices for patient- and family-centered bedside rounds. Tips on bedside rounding were included in a general medicine rotation guidebook made available to residents and attendings. At the beginning of each post-intervention general medicine rotation, attendings and residents attended brief orientation sessions to review the new daily schedule, have interdisci- plinary huddles, and share expectations for patient- and family-centered bedside rounds. On the general medicine units, new medical directors were hired to partner with existing nursing directors to support adoption of the workflows. Last, an interdisciplinary leadership team was formed to support the care redesign efforts. This team started meeting every 2 weeks.

\section{Study Design}

We used a pre-post analysis to study the effects of care redesign. Analysis was performed at the same time of year for 2 consecutive years to control for the stage of training and experience of the housestaff. TMA was performed by trained medical students using computer tablets linked to a customized Microsoft Access database form (Redmond, Washington). The form and the database were designed with specific buttons that, when pressed, recorded the time of particular events, such as the coming and going of each participant, the location of rounds, and the beginning and the end of rounding encounters with a patient. One research assistant using an Access entry form was able to dynamically track all events in real time, as they occurred. We collected data on 4 teams at baseline and 5 teams after the intervention. Each of the 4 baseline teams was followed for 4 consecutive weekdays-16 rounds total, April-June 2013-to capture the 4-day call cycle. Each of the 5 post-intervention teams was followed for 5 consecutive weekdays -25 rounds total, April-June 2014-to capture the 5-day cycle. (Because of technical difficulties, data from 1 rounding session were not captured.) For inclusion in the statistical analyses, TMA captured 166 on-service patients before the intervention and 304 afterward. Off-service patients, those with an attending other than the team attending, were excluded because their rounds were conducted separately.

We examined 2 primary outcomes, the proportion of time each clinical team member was present on rounds and the proportion of bedside rounding time. Secondary outcomes were round duration, rounding time per patient, and total non-patient time per rounding session (total rounding time minus total patient time).

\section{Statistical Analysis}

TMA data were organized in an Access database and analyzed with SAS Version 9.3 (SAS Institute, Cary, North Carolina). We analyzed the data by round session as well as by patient.

Data are presented as means with standard deviations, medians with interquartile ranges, and proportions, as appropriate. For analyses by round session, we used unadjusted linear regression; for patient-level analyses, we used general estimating equations to adjust for clustering of patients within each session; for nurse presence during any part of a round by patient, we used a $\chi^{2}$ test. Total non-patient time per round session was compared with use of patient-clustered general estimating equations using a $\gamma$ distribution to account for the non-normality of the data. 
TABLE 1. Demographics of Patients on General Medical Service Before and After Implementation of Data Collection

\begin{tabular}{|c|c|c|c|}
\hline Characteristic & $\begin{array}{c}\text { Before Implementation } \\
\text { (April-June 2013) } \\
\qquad \mathrm{N}=820\end{array}$ & $\begin{array}{l}\text { After Implementation } \\
\text { (April-June 2014) } \\
\qquad \mathrm{N}=780\end{array}$ & $P$ \\
\hline Mean (SD) age, y & $58.8(19.7)$ & $58.7(20.1)$ & 0.89 \\
\hline Median (IQR) length of stay, d & $4(2,6)$ & $4(2,7)$ & 0.24 \\
\hline Mean (SD) Elixhauser comorbidity score & $8.1(8.7)$ & $8.2(8.9)$ & 0.84 \\
\hline Female, n (\%) & $457(55.7 \%)$ & $435(55.8 \%)$ & $>0.99$ \\
\hline Race/ethnicity, n (\%) & & & 0.92 \\
\hline White & $524(64 \%)$ & $494(63 \%)$ & \\
\hline Black & $179(22 \%)$ & $163(21 \%)$ & \\
\hline Hispanic/Latino & $68(8 \%)$ & $73(9 \%)$ & \\
\hline Other & $25(3 \%)$ & $27(3 \%)$ & \\
\hline Declined/unavailable & $24(3 \%)$ & $23(3 \%)$ & \\
\hline Language, n (\%) & & & 0.25 \\
\hline English & $754(92 \%)$ & $704(90 \%)$ & \\
\hline Other & $66(8 \%)$ & $76(10 \%)$ & \\
\hline Admission source, $n(\%)$ & & & 0.049 \\
\hline Other facility & $206(25 \%)$ & $190(24 \%)$ & \\
\hline Home & $561(68 \%)$ & $560(72 \%)$ & \\
\hline Clinic & $53(6 \%)$ & $30(4 \%)$ & \\
\hline Marital status & & & 0.81 \\
\hline Married or living as married & $299(37 \%)$ & $273(35 \%)$ & \\
\hline Divorced, separated, or widowed & 197 (24\%) & $187(24 \%)$ & \\
\hline Single, never married & $316(39 \%)$ & $311(40 \%)$ & \\
\hline
\end{tabular}

\section{RESULTS}

\section{Patient and Care Team Characteristics}

Over the first year of the initiative, $85 \%$ of a team's patients were on their assigned unit, and $87 \%$ of a unit's patients were with the assigned team. Census numbers were 10.4 patients per general medicine team in April-June 2013 and 12.7 patients per team in April-June 2014, a 22\% increase after care redesign. There were no statistically significant differences in patient characteristics, including age, sex, race, language, admission source, and comorbidity measure (Elixhauser score), between the pre-intervention and post-intervention study periods, except for a slightly higher proportion of patients admitted from home and fewer patients admitted directly from clinic (Table 1 ).

\section{Primary Outcomes}

Mean proportion of time the nurse was present on rounds per round session increased significantly $(P<0.001)$, from $24.1 \%$ to $67.8 \%$ (Figure $1 \mathrm{~A}$, Table 2 ). For individual patient encounters, the increased overall nursing presence was attributable to having more nurses on rounds and having nurses present for a larger proportion of individual rounding encounters (Figure 1B, Table 2). Nurses were present for at least some part of rounds for $53 \%$ of patients before the intervention and $93 \%$ afterward $(P<0.001)$. Mean proportion of round time by each of the 2 interns on each team decreased from $59.6 \%$ to $49.6 \%(P=0.007)$.

Total bedside rounding time increased significantly $(P<$
0.001 ), from $39.9 \%$ before the intervention to $55.8 \%$ afterward (Table 2). Meanwhile, percentage of rounding time spent on the unit but outside patient rooms decreased significantly $(P=0.004)$, from $55.2 \%$ to $42.2 \%$, as did rounding time on a unit completely different from the patient's (4.9\% before intervention, $2.0 \%$ afterward; $P=0.03$ ). Again, patient-level results were similar (Figure 2, Table 2), but the decreased time spent on the unit, outside the patient rooms, was not significant.

\section{Secondary Outcomes}

Total rounding time decreased significantly, from a mean of 182 minutes ( 3.0 hours) at baseline to a mean of $146 \mathrm{~min}$ utes (2.4 hours) after the intervention, despite the higher post-intervention census. (When adjusted for patient census, the difference increased from 35.5 to 53.8 minutes; Table 2.) Mean rounding time per patient decreased significantly, from 14.7 minutes at baseline to 10.5 minutes after the intervention. For newly admitted patients, mean rounding time per patient decreased from 30.0 minutes before implementation to 16.3 minutes afterward. Mean rounding time also decreased, though much less, for subsequent-day patients (Table 2). For both new and existing patients, the decrease in rounding time largely was a reduction in time spent rounding outside patient rooms, with minimal impact on bedside time (Table 2). Mean time nurses were present during a patient's rounds increased significantly, from 4.5 to 8.0 minutes (Table 2). Total nurse rounding time increased 
from 45.1 minutes per session to $98.8 \mathrm{~min}$ utes. Rounding time not related to patient discussion or evaluation decreased from 22.7 minutes per session to 13.3 minutes $(P=0.003)$.

\section{DISCUSSION}

TMA of our care redesign initiative showed that this multipronged intervention, which included team regionalization, encouragement of bedside rounding with nurses, call structure changes, and attendings' reading of admission notes before rounds, resulted in an increased proportion of rounding time spent with patients and an increased proportion of time nurses were present on rounds. Secondarily, round duration decreased even as patient census increased.

Regionalized teams have been found to improve interdisciplinary communication. ${ }^{1}$ The present study elaborates on that finding by demonstrating a dramatic increase in nursing presence on rounds, likely resulting from the unit's use of rounding schedules and nurses' prioritization of rounding orders, both of which were made possible by geographic co-localization. Other research has noted that one of the most significant barriers to interdisciplinary rounds is difficulty coordinating the start times of physician/nurse bedside rounding encounters. The system we have studied directly addresses this difficulty. ${ }^{9}$ Of note, nursing presence on rounds is necessary but not sufficient for true physician-nurse collaboration and effective communication, ${ }^{1}$ as reflected in a separate study of the intervention showing no significant difference in the concordance of the patient care plan between nurses and physicians before and after regionalization. ${ }^{12}$ Additional interventions may be needed to ensure that communication during bedside rounds is effective.

Our regionalized teams spent a significantly higher proportion of rounding time bedside, likely because of a cultural shift in expectations and the increased convenience of seeing patients on the team's unit. Nevertheless, bedside time was not $100 \%$. Structural barriers (eg, patients off-unit for dialysis) and cultural barriers likely contributed to the less than full adoption of bedside rounding. As described previously, cultural barriers to bedside rounding include trainees' anxiety about being questioned in front of patients, the desire to freely exchange academic ideas in a conference room, and attendings' doubts
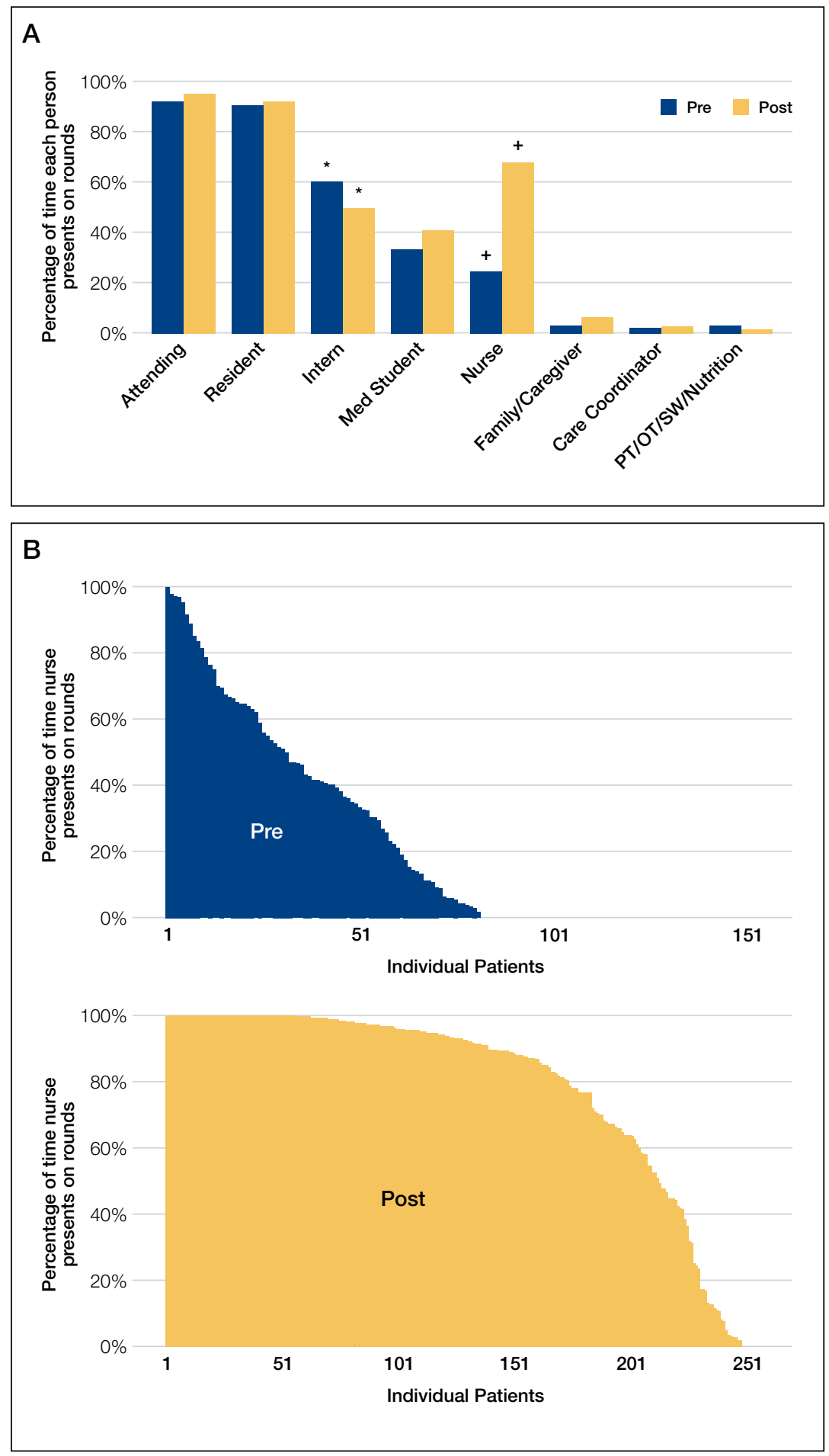

FIG. 1. Staff presence on rounds. (A) Proportion of time each care team member was present on rounds before and after intervention. Symbols indicate statistically significant differences $\left({ }^{\star} P<0.01 ;{ }^{+} P\right.$ $<0.001$ ) before and after intervention for intern and nurse. NOTE: Abbreviations: OT, occupational therapist; PT, physical therapist; SW, social worker. (B) Percentage of time nurse was present on rounds by individual patient before and after intervention. Each unit on $\mathrm{x}$-axis represents patient's rounding time, with shaded vertical column denoting percentage of time nurse was present for that patient's rounds. For example, during pre-intervention period, nurse was present for $100 \%$ of rounds for 1 patient, for more than $80 \%$ of rounds for about 10 of 166 patients, and for no rounds for about half of all patients. In contrast, during post-intervention period, nurse was present for $100 \%$ of rounds for about 60 of 304 patients, for more than $80 \%$ of rounds for about 160 patients, and for no rounds for 50 patients. 
TABLE 2. Primary and Secondary Outcomes

\begin{tabular}{|c|c|c|c|c|}
\hline Outcome & $\begin{array}{l}\text { Before Implementation, mean } \\
\text { (SD) }\end{array}$ & $\begin{array}{l}\text { After Implementation, } \\
\text { mean (SD) }\end{array}$ & $\begin{array}{c}\text { Adjusted Difference } \\
(95 \% \mathrm{Cl})^{\mathrm{a}}\end{array}$ & $P$ \\
\hline Analysis by rounding session & $N=16$ & $N=25$ & & \\
\hline Proportion of time nurse present on rounds & $24.1 \%(10.8 \%)$ & $67.8 \%(13.0 \%)$ & $43.8 \%$ (36.2\% to $51.3 \%)$ & $<0.001$ \\
\hline Total rounding time, min & $182(53.2)$ & $146(30.0)$ & $53.8(27.6 \text { to } 80.0)^{b}$ & $<0.001$ \\
\hline Total nurse rounding time, min & $45.1(26.1)$ & $98.8(25.3)$ & 53.7 (36.9 to 70.4$)$ & $<0.001$ \\
\hline Analysis by patient & $N=166$ & $N=304$ & & \\
\hline Proportion of time rounding bedside & $41.4 \%(27.6 \%)$ & $53.2 \%(37.0 \%)$ & $11.9 \%(2.0 \%$ to $21.8 \%)$ & 0.02 \\
\hline Total rounding time per patient, min & $14.7(11.2)$ & $10.5(6.4)$ & $4.1(2.5$ to 5.8$)$ & $<0.001$ \\
\hline New admissions & $30.0(10.7)$ & $16.3(7.4)$ & 13.8 (9.5 to 18.1$)$ & $<0.001$ \\
\hline Subsequent-day patients & $11.9(8.8)$ & $9.3(5.4)$ & $2.6(1.1$ to 4.1$)$ & $<0.001$ \\
\hline \multicolumn{5}{|l|}{ Rounding time per patient by location, min } \\
\hline New admissions_-bedside & $12.2(6.9)$ & $11.7(5.4)$ & $0.5(-2.2$ to 3.3$)$ & 0.71 \\
\hline $\begin{array}{l}{ }^{2} \text { For analyses by patient, clustered by patient within ead } \\
\text { 'AAdjusted for number of patients. } \\
\text { NOTE: Abbreviations: Cl, confidence interval; SD, stand }\end{array}$ & ession. & & & \\
\hline
\end{tabular}

about their bedside teaching ability. ${ }^{1,9,13}$ Bedside rounds provide an important opportunity to apply the principles of patient-and family-centered care, including promotion of dignity and respect, information sharing, and collaboration. Thus, overcoming the concerns of housestaff and attendings and helping them feel prepared for bedside rounds can benefit the patient experience. More attention should be given to these practices as these types of interventions are implemented at Brigham and Women's Hospital and elsewhere. ${ }^{1,13-15}$

Another primary concern about interdisciplinary bedside rounding is the perception that it takes more time. ${ }^{9}$ Therefore, it was important for us to measure round duration as a balancing measure to be considered for our intervention. Fortunately, we found round duration decreased with regionalization and encouragement of bedside rounding. This decrease was driven largely by a significant decrease in mean rounding time per new patient, which may be attributable at least in part to setting expectations that attendings and residents will read admission notes before rounds and that interns will summarize rather than recount information from admission notes. However, we also found rounding time decreases for subsequent-day patients, suggesting an underlying time savings. Spending a larger proportion of time bedside may therefore result in more efficient rounds. Bedside presentations can reduce redundancies, such as discussing a patient's case outside his or her room and subsequently walking in and going over much of the same information with the patient. Our model de-emphasizes data transfer in favor of discussion of care plans. There was also a decrease in non-patient time, likely reflecting reduced transit time for regionalized teams. This decrease aligns with a recent finding that bedside rounding was at least as efficient as rounding outside the room. ${ }^{16}$
Of note, though a larger percentage of time was spent bedside after implementation of the care redesign, the absolute amount of bedside time did not change significantly. Our data showed that, even with shorter rounds, the same amount of absolute time can be spent bedside, face to face with the patient, by increasing the proportion of bedside rounding time. In other words, teams on average did not spend more time with patients, though the content and the structure of those encounters may have changed. This finding may be attributable to eliminating redundancy, forgoing the outside-the-room discussion, and thus the largest time reductions were realized there. In addition, teams incompletely adopted beside rounds, as reflected in the data. We expect that, with more complete adoption, an even larger proportion of time will be spent bedside, and absolute time bedside might increase as a result.

An unexpected result of the care redesign was that interns' proportion of rounding time decreased after the intervention. This decrease most likely is attributable to interns' being less likely to participate in rounds for a co-intern's patient, and to their staying outside that patient's room to give themselves more time to advance the care of their own patients. Before the intervention, when more rounding time was spent outside patient rooms, interns were more likely to join rounds for their co-intern's patients because they could easily break away, as needed, to continue care of their own patients. The resident is now encouraged to use the morning huddle to identify which patients likely have the most educational value, and both interns are expected to join the bedside rounds for these patients.

This study had a few limitations. First, the pre-post design made it difficult to exclude the possibility that other temporal changes may have affected outcomes, though we did 
account for time-of-year effects by aligning our data-collection phases. In addition, the authors, including the director of the general medical service, are unaware of any co-interventions during the study period. Second, the multipronged intervention included care team regionalization, encouragement of bedside rounding with nurses, call structure changes (from 4 days to daily admitting), and attendings' reading of admission notes before rounds. Thus, parsing which component(s) contributed to the results was difficult, though all the changes instituted likely were necessary for system redesign. For example, regionalization of clinicians to unit-based teams was made possible by switching to a daily admitting system.

Time that team members spent preparing for rounds was not recorded before or after the intervention. Thus, the decrease in total rounding time could have been accompanied by an increase in time spent preparing for rounds. However, admission notes were available in our electronic medical record before and after the intervention, and most residents and attendings were already reading them pre-intervention. After the intervention, pre-round note reading was more clearly defined as an expectation, and we were able to set the expectation that interns should use their presentations to summarize rather than recount information. In addition, in the post-intervention period, we did not include time spent preparing rounding orders; as already noted, however, preparation took only 5 minutes per day. Also, we did not analyze the content or the quality of the discussion on rounds, but simply recorded who was present where and when. Regarding the effect of the intervention on patient care, results were mixed. As reported in 2016, we saw no difference in frequency of adverse events with this intervention. ${ }^{12}$ However, a more sensitive measure of adverse events—used in a study on handoffs—showed our regionalization efforts had an additive effect on reducing overnight adverse events. ${ }^{17}$

Researchers should now focus on the effects of care redesign on clinical outcomes, interdisciplinary care team communication, patient engagement and satisfaction, provider opinions of communication, workflow, patient care, and housestaff education. Our methodology can be used as a model to link structure, process, and outcome related to rounds and thereby better understand how best to optimize patient care and efficiency. Additional studies are needed to analyze the content of rounds and their association with patient and educational outcomes. Last, it will be important to conduct a study to see if the effects we have identified can be sustained. Such a study is already under way.
In conclusion, creating regionalized care teams and encouraging focused bedside rounds increased the proportion of bedside time and the presence of nurses on rounds. Rounds were shorter despite higher patient census. TMA revealed that regionalized care teams and bedside rounding at a large academic hospital are feasible, and are useful in establishing the necessary structures for increasing physician-nurse and provider-patient interactions.

\section{Acknowledgments}

The authors acknowledge Dr. Stan Ashley, Dr. Jacqueline Somerville, and Sheila Harris for their support of the regionalization initiative.

Disclosures: Dr. Schnipper received funding from Sanofi-aventis to conduct an investigator-initiated study to implement and evaluate a multi-faceted intervention to improve transitions of care in patients discharged home on insulin. The study was also supported by funding from the Marshall A. Wolf Medical Education Fund, Brigham and Women's Hospital, and Dr. Stan Ashley, Chief Medical Officer, Brigham and Women's Hospital. Some of the content of this article was orally presented at the annual meeting of the Society of Hospital Medicine; March 29-April 1, 2015; National Harbor, MD. 


\section{References}

1. Crumlish CM, Yialamas MA, McMahon GT. Quantification of bedside teaching by an academic hospitalist group. J Hosp Med. 2009;4(5):304-307.

2. Gonzalo JD, Masters PA, Simons RJ, Chuang CH. Attending rounds and bedside case presentations: medical student and medicine resident experiences and attitudes. Teach Learn Med. 2009;21(2):105-110.

3. Elliot DL, Hickam DH. Attending rounds on in-patient units: differences between medical and non-medical services. Med Educ. 1993;27(6):503-508.

4. Payson HE, Barchas JD. A time study of medical teaching rounds. N Engl J Med. 1965;273(27):1468-1471.

5. Tremonti LP, Biddle WB. Teaching behaviors of residents and faculty members. $J$ Med Educ. 1982;57(11):854-859.

6. Miller M, Johnson B, Greene HL, Baier M, Nowlin S. An observational study of attending rounds. J Gen Intern Med. 1992;7(6):646-648.

7. Collins GF, Cassie JM, Daggett CJ. The role of the attending physician in clinical training. J Med Educ. 1978;53(5):429-431.

8. Ward DR, Ghali WA, Graham A, Lemaire JB. A real-time locating system observes physician time-motion patterns during walk-rounds: a pilot study. BMC Med Educ. 2014;14:37

9. Gonzalo JD, Kuperman E, Lehman E, Haidet P. Bedside interprofessional rounds: perceptions of benefits and barriers by internal medicine nursing staff, attending physicians, and housestaff physicians. J Hosp Med. 2014;9(10):646-651.

10. Stickrath C, Noble M, Prochazka A, et al. Attending rounds in the current era: what is and is not happening. JAMA Intern Med. 2013;173(12):1084-1089.

11. Boxer R, Vitale M, Gershanik EF, et al. 5th time's a charm: creation of unit-based care teams in a high occupancy hospital [abstract]. J Hosp Med. 2015;10(suppl 2).

12. Mueller SK, Schnipper JL, Giannelli K, Roy CL, Boxer R. Impact of regionalized care on concordance of plan and preventable adverse events on general medicine services. J Hosp Med. 2016;11(9):620-627.

13. Chauke HL, Pattinson RC. Ward rounds-bedside or conference room? S Afr Med J. 2006;96(5):398-400.

14. Wang-Cheng RM, Barnas GP, Sigmann P, Riendl PA, Young MJ. Bedside case presentations: why patients like them but learners don't. J Gen Intern Med. 1989;4(4):284-287.

15. Lehmann LS, Brancati FL, Chen MC, Roter D, Dobs AS. The effect of bedside case presentations on patients' perceptions of their medical care. $N$ Engl J Med. 1997;336(16):1150-1155.

16. Gonzalo JD, Chuang $\mathrm{CH}$, Huang $\mathrm{G}$, Smith C. The return of bedside rounds: an educational intervention. J Gen Intern Med. 2010;25(8):792-798.

17. Mueller SK, Yoon C, Schnipper JL. Association of a web-based handoff tool with rates of medical errors. JAMA Intern Med. 2016;176(9):1400-1402. 